\title{
CONVEXITY OF THE IMAGE OF A QUADRATIC MAP VIA THE RELATIVE ENTROPY DISTANCE
}

\author{
AleXANDER BARVINOK
}

May 2013

\begin{abstract}
Let $\psi: \mathbb{R}^{n} \longrightarrow \mathbb{R}^{k}$ be a map defined by $k$ positive definite quadratic forms on $\mathbb{R}^{n}$. We prove that the relative entropy (Kullback-Leibler) distance from the convex hull of the image of $\psi$ to the image of $\psi$ is bounded above by an absolute constant. More precisely, we prove that for every point $a=\left(a_{1}, \ldots, a_{k}\right)$ in the convex hull of the image of $\psi$ such that $a_{1}+\ldots+a_{k}=1$ there is a point $b=\left(b_{1}, \ldots, b_{k}\right)$ in the image of $\psi$ such that $b_{1}+\ldots+b_{k}=1$ and such that $\sum_{i=1}^{k} a_{i} \ln \left(a_{i} / b_{i}\right)<4.8$. Similarly, we prove that for any integer $m$ one can choose a convex combination $b$ of at most $m$ points from the image of $\psi$ such that $\sum_{i=1}^{k} a_{i} \ln \left(a_{i} / b_{i}\right)<15 / \sqrt{m}$.
\end{abstract}

\section{INTRODUCTION}

Let $q_{1}, \ldots, q_{k}: \mathbb{R}^{n} \longrightarrow \mathbb{R}$ be quadratic forms and let $\psi: \mathbb{R}^{n} \longrightarrow \mathbb{R}^{k}$ be the corresponding quadratic map,

$$
\psi(x)=\left(q_{1}(x), \ldots, q_{k}(x)\right) .
$$

We are interested in the convex properties of the image $\psi\left(\mathbb{R}^{n}\right) \subset \mathbb{R}^{k}$. The image is clearly convex when $k=1$ and by the Dines Theorem it is convex when $k=2$ (this and related facts can be found, for example, in Sections II.12-14 of [Ba02] or in [PT07]). The image is not necessarily convex for $k \geq 3$, though it remains convex for $k=3$ if some linear combination of the forms $q_{1}, q_{2}$ and $q_{3}$ is positive definite.

In this paper, we show that the image $\psi\left(\mathbb{R}^{n}\right)$ is close to its own convex hull $\operatorname{conv}\left(\psi\left(\mathbb{R}^{n}\right)\right)$ in some information-theoretic sense.

Let

$$
a=\left(a_{1}, \ldots, a_{k}\right) \quad \text { and } \quad b=\left(b_{1}, \ldots, b_{k}\right)
$$

1991 Mathematics Subject Classification. 52A27, 52A20, 52B55, 90C22, 62B20.

Key words and phrases. Kullback - Leibler distance, relative entropy, quadratic convexity, positive semidefinite programming, Johnson - Lindenstrauss Lemma, Gaussian measure.

This research was partially supported by NSF Grant DMS 0856640. 
be two positive vectors such that

$$
\sum_{i=1}^{k} a_{i}=\sum_{i=1}^{k} b_{i}=1
$$

We interpret $a$ and $b$ as probability distributions and define the relative entropy of $a$ with respect to $b$ as

$$
D(a \| b)=\sum_{i=1}^{k} a_{i} \ln \left(\frac{a_{i}}{b_{i}}\right) .
$$

The quantity $D(a \| b)$ is also known as the Kullback - Leibler distance from $a$ to $b$ (although, generally speaking, $D(a \| b) \neq D(b \| a)$ and the triangle inequality does not hold). In particular, $D(a \| b) \geq 0$ with equality if and only if $a=b$, see for example, [CT06].

We prove that with respect to the Kullback - Leibler distance, the image $\psi\left(\mathbb{R}^{n}\right)$ of a quadratic map is reasonably close to its own convex hull conv $\left(\psi\left(\mathbb{R}^{n}\right)\right)$.

(1.1) Theorem. Let $q_{1}, \ldots, q_{k}: \mathbb{R}^{n} \longrightarrow \mathbb{R}$ be positive definite quadratic forms and let $\psi: \mathbb{R}^{n} \longrightarrow \mathbb{R}^{k}$ be the corresponding map,

$$
\psi(x)=\left(q_{1}(x), \ldots, q_{k}(x)\right) .
$$

Let $a \in \operatorname{conv}\left(\psi\left(\mathbb{R}^{n}\right)\right)$ be a point, $a=\left(a_{1}, \ldots, a_{k}\right)$, such that $a_{1}+\ldots+a_{k}=1$. Then there exists a point $b \in \psi\left(\mathbb{R}^{n}\right), b=\left(b_{1}, \ldots, b_{k}\right)$, such that $b_{1}+\ldots+b_{k}=1$ and

$$
\sum_{i=1}^{k} a_{i} \ln \left(\frac{a_{i}}{b_{i}}\right) \leq \beta
$$

for some absolute constant $\beta>0$. One can choose, for example, $\beta=4.8$.

We have undertaken some effort to optimize the constant $\beta$, but its optimal value is not known at the moment and it would be interesting to find it.

Loosely speaking, Theorem 1.1 asserts that replacing the image of $\psi$ by its convex hull leads to only a constant loss of information. The technique of semidefinite programming is based on replacing computationally intractable systems of quadratic equations and inequalities over the reals by computationally tractable systems of linear equations and inequalities in positive semidefinite matrices. This procedure is known as relaxation, see for example, [Tu10]. The success of relaxation depends on the convex properties of the underlying quadratic maps, see [PT07]. Speaking even more loosely, one can speculate that the constant bound on the information loss in Theorem 1.1 explains the success of semidefinite programming.

We also prove the following extension of Theorem 1.1. 
(1.2) Theorem. Let $q_{1}, \ldots, q_{k}: \mathbb{R}^{n} \longrightarrow \mathbb{R}$ be positive definite quadratic forms and let $\psi: \mathbb{R}^{n} \longrightarrow \mathbb{R}^{k}$ be the corresponding map,

$$
\psi(x)=\left(q_{1}(x), \ldots, q_{k}(x)\right) .
$$

Let $a \in \operatorname{conv}\left(\psi\left(\mathbb{R}^{n}\right)\right)$ be a point, $a=\left(a_{1}, \ldots, a_{k}\right)$, such that $a_{1}+\ldots+a_{k}=1$. Then, for any positive integer $m$, there exists a point $b=\left(b_{1}, \ldots, b_{k}\right)$, such that $b_{1}+\ldots+b_{k}=1$, the point $b$ is a convex combination of at most $m$ points of $\psi\left(\mathbb{R}^{n}\right)$ and

$$
\sum_{i=1}^{k} a_{i} \ln \left(\frac{a_{i}}{b_{i}}\right)<\frac{15}{\sqrt{m}}
$$

We note a useful inequality

$$
D(a \| b)=\sum_{i=1}^{k} a_{i} \ln \left(\frac{a_{i}}{b_{i}}\right) \geq \frac{1}{2 \ln 2}\left(\sum_{i=1}^{k}\left|a_{i}-b_{i}\right|\right)^{2},
$$

see, for example, Section 11.6 of [CT06]. The Approximate Carathéodory Theorem of Maurey (see [Pi81] and Section I.3 of [Ve+]) states that if $X$ is any set of points in the standard simplex

$$
\sum_{i=1}^{k} x_{i}=1 \quad \text { and } \quad x_{1}, \ldots, x_{k} \geq 0
$$

in $\mathbb{R}^{k}$ then any point $a \in \operatorname{conv}(X)$ can be approximated within error of $1 / \sqrt{m}$ by a convex combination of $m$ points of $X$ in the $\ell^{2}$ (Euclidean) norm. Theorem 1.2 asserts that if $X$ is the image of a quadratic map then one can get a similar approximation in the $\ell^{1}$ norm.

The Johnson - Lindenstrauss Lemma implies that for any $\epsilon>0$, if one chooses $m=O\left(\epsilon^{-2} \ln k\right)$ in Theorem 1.2 then one can ensure that

$$
\left|\ln \frac{a_{i}}{b_{i}}\right| \leq \epsilon \quad \text { for } \quad i=1, \ldots, k,
$$

see, for example, Sections V.5-6 of [Ba02] and [Ma08]. Theorem 1.2 asserts that if we measure the Kullback - Leibler distance, then the dependence on the number $k$ of quadratic forms can be removed so that $m=O\left(\epsilon^{-2}\right)$ and

$$
D(a \| b)=\sum_{i=1}^{k} a_{i} \ln \left(\frac{a_{i}}{b_{i}}\right) \leq \epsilon .
$$

In the rest of the paper, we prove Theorems 1.1 and 1.2. In Section 2, we establish some general results on the distribution of values of a positive semidefinite 
quadratic form with respect to the Gaussian probability measure in $\mathbb{R}^{n}$. In Section 3 , we consider the problem of maximizing a convex combination of logarithms of positive semidefinite quadratic forms on the unit sphere. We prove that its straightforward positive semidefinite relaxation produces a relative error bounded by an absolute constant. In Section 4, we complete the proof of Theorem 1.1. The proof of Theorem 1.2 given in Section 5 is a straightforward modification of our proof of Theorem 1.1.

\section{QuAdratic Forms and the GaUssian MeAsure}

Un this section, we prove the following main result.

(2.1) Lemma. Let us fix in $\mathbb{R}^{n}$ the standard Gaussian probability measure $\mu_{n}$ with density

$$
\frac{1}{(2 \pi)^{n / 2}} e^{-\|x\|^{2} / 2}
$$

Let $q: \mathbb{R}^{n} \longrightarrow \mathbb{R}$ be a positive semidefinite quadratic form such that

$$
\mathbf{E} q=1 \text {. }
$$

Then

(1) We have

$$
\mathbf{E}|\ln q|<2.75
$$

(2) For $t \geq 1$ let us define

$$
\phi(t)=\min _{\alpha \geq 1} \frac{2^{\alpha}}{t^{\alpha} \sqrt{\pi}} \Gamma\left(\alpha+\frac{1}{2}\right) .
$$

Then

$$
\mathbf{P}(x: q(x) \geq t) \leq \phi(t) \quad \text { for all } t \geq 1 \text {. }
$$

Proof. Part (1) is essentially proved in [Ba99] but we present its proof here for completeness. We have

$$
\mathbf{E}|\ln q| \leq\left(\mathbf{E} \ln ^{2} q\right)^{1 / 2} .
$$

We can write

$$
q(x)=\sum_{i=1}^{n} \lambda_{i} x_{i}^{2} \quad \text { for } \quad x=\left(x_{1}, \ldots, x_{n}\right)
$$

in some orthonormal basis of $\mathbb{R}^{n}$. Since

$$
\mathbf{E} q=\mathbf{E} x_{i}^{2}=1 \quad \text { for } \quad i=1, \ldots, n
$$


we have

$$
\sum_{i=1}^{n} \lambda_{i}=1 \quad \text { and also } \quad \lambda_{i} \geq 0 \quad \text { for } \quad i=1, \ldots, n \text {. }
$$

Let

$$
Y=\left\{x \in \mathbb{R}^{n}: q(x) \leq 1\right\}
$$

By the concavity of the logarithm,

$$
\ln \left(\sum_{i=1}^{n} \lambda_{i} x_{i}^{2}\right) \geq \sum_{i=1}^{n} \lambda_{i} \ln x_{i}^{2}
$$

Since $\ln q(x)<0$ for all $x \in Y$, using (2.1.2) and the convexity of the function $t \longmapsto t^{2}$, we conclude that

$$
\begin{aligned}
\int_{Y} \ln ^{2} q(x) d \mu_{n}(x) & \leq \int_{Y}\left(\sum_{i=1}^{n} \lambda_{i} \ln x_{i}^{2}\right)^{2} d \mu_{n}(x) \\
& \leq \int_{Y}\left(\sum_{i=1}^{n} \lambda_{i} \ln ^{2} x_{i}^{2}\right) d \mu_{n}(x) \leq \int_{\mathbb{R}^{n}} \ln ^{2} x_{1}^{2} d \mu_{n}(x) \\
& =\frac{8}{\sqrt{2 \pi}} \int_{0}^{+\infty}\left(\ln ^{2} x\right) e^{-x^{2} / 2} d x<6.55 .
\end{aligned}
$$

On the other hand, since $\ln t \leq \sqrt{t}$ for $t \geq 1$, we conclude that

$$
\int_{\mathbb{R}^{n} \backslash Y} \ln ^{2} q(x) d \mu_{n}(x) \leq \int_{\mathbb{R}^{n} \backslash Y} q(x) d \mu_{n}(x) \leq \int_{\mathbb{R}^{n}} q(x) d \mu_{n}(x)=1 .
$$

Therefore,

$$
\mathbf{E} \ln ^{2} q<6.55+1=7.55 \text { and } \mathbf{E}|\ln q|<\sqrt{7.55}<2.75,
$$

which proves Part (1).

Let us choose any $\alpha \geq 1$. Applying the Markov inequality, we get

$$
\mathbf{P}(x: q(x) \geq t)=\mathbf{P}\left(x: q^{\alpha}(x) \geq t^{\alpha}\right) \leq t^{-\alpha} \mathbf{E} q^{\alpha} .
$$

Writing $q$ as in (2.1.1) and using (2.1.2) and the convexity of the function $t \longmapsto t^{\alpha}$, we obtain

$$
\begin{aligned}
\mathbf{E} q^{\alpha} & =\mathbf{E}\left(\sum_{i=1}^{n} \lambda_{i} x_{i}^{2}\right)^{\alpha} \leq \sum_{i=1}^{k} \lambda_{i} \mathbf{E}\left(x_{i}^{2}\right)^{\alpha} \\
& =\frac{2}{\sqrt{2 \pi}} \int_{0}^{+\infty} x^{2 \alpha} e^{-x^{2} / 2} d x=\frac{2^{\alpha}}{\sqrt{\pi}} \Gamma\left(\alpha+\frac{1}{2}\right),
\end{aligned}
$$


from which the proof of Part (2) follows.

(2.2) Remark. The exact upper bound in Part (1) is not known to the author, though it looks plausible that it is attained on forms of rank 1 and hence is equal to

$$
\frac{4}{\sqrt{2 \pi}} \int_{0}^{+\infty}|\ln x| e^{-x^{2} / 2} d x \approx 1.76 .
$$

\section{An optimization PRoblem on the SPhere}

(3.1) Notation. We consider the space $\operatorname{Sym}_{n}$ of $n \times n$ symmetric matrices endowed with standard inner product

$$
\langle A, B\rangle=\sum_{i, j=1}^{n} a_{i j} b_{i j}=\operatorname{trace}(A B),
$$

where $A=\left(a_{i j}\right)$ and $B=\left(b_{i j}\right)$. For a vector $x \in \mathbb{R}^{n}, x=\left(x_{1}, \ldots, x_{n}\right)$, we define a symmetric matrix $X=x \otimes x, X=\left(x_{i j}\right)$, by $x_{i j}=x_{i} x_{j}$. Thus a quadratic form $q$ with matrix $Q$ can be written as

$$
q(x)=\langle Q, x \otimes x\rangle \quad \text { for all } \quad x \in \mathbb{R}^{n} .
$$

We write $X \succeq 0$ to say that $X$ is positive semidefinite and $X \succ 0$ to say that $X$ is positive definite.

In $\mathbb{R}^{n}$, we consider the standard inner product

$$
\langle x, y\rangle=\sum_{i=1}^{n} x_{i} y_{i} \quad \text { where } \quad x=\left(x_{1}, \ldots, x_{n}\right) \quad \text { and } \quad y=\left(y_{1}, \ldots, y_{n}\right)
$$

the corresponding norm

$$
\|x\|=\sqrt{\langle x, x\rangle}
$$

and the unit sphere

$$
\mathbb{S}^{n-1}=\left\{x \in \mathbb{R}^{n}: \quad\|x\|=1\right\} .
$$

In this section, we prove the following main result.

(3.2) Theorem. Let $\alpha_{1}, \ldots, \alpha_{k}$ be non-negative reals such that $\alpha_{1}+\ldots+\alpha_{k}=1$, let $Q_{1}, \ldots, Q_{k}$ be $n \times n$ positive definite matrices and let $q_{1}, \ldots, q_{k}: \mathbb{R}^{n} \longrightarrow \mathbb{R}$ be the corresponding quadratic forms,

$$
q_{i}(x)=\left\langle Q_{i}, x \otimes x\right\rangle \quad \text { for } \quad i=1, \ldots, k .
$$

Then

$$
\max _{x \in \mathbb{S}^{n-1}} \sum_{i=1}^{k} \alpha_{i} \ln q_{i}(x) \leq \max _{\substack{X \succeq 0 \\ \operatorname{trace}(X)=1}} \sum_{i=1}^{k} \alpha_{i} \ln \left\langle Q_{i}, X\right\rangle \leq \beta+\max _{x \in \mathbb{S}^{n-1}} \sum_{i=1}^{k} \alpha_{i} \ln q_{i}(x),
$$


where $\beta>0$ is an absolute constant. One can choose $\beta=4.8$.

Proof. For $x \in \mathbb{S}^{n-1}$ the matrix $X=x \otimes x$ satisfies the constraints $X \succeq 0$ and $\operatorname{trace}(X)=1$. Hence the first inequality holds.

Let $A$ be a matrix where the maximum value of the function

$$
X \longmapsto \sum_{i=1}^{k} \alpha_{i} \ln \left\langle Q_{i}, X\right\rangle
$$

is attained on the set $X$ of positive semidefinite matrices of trace 1. Rescaling $Q_{i} \longrightarrow \tau_{i} Q_{i}$ for some positive $\tau_{1}, \ldots, \tau_{k}$ if necessary, we may assume that $\left\langle Q_{i}, A\right\rangle=$ 1 for $i=1, \ldots, k$ and hence

$$
\max _{\substack{X \succeq 0 \\ \operatorname{trace}(X)=1}} \sum_{i=1}^{k} \alpha_{i} \ln \left\langle Q_{i}, X\right\rangle=\sum_{i=1}^{k} \alpha_{i} \ln \left\langle Q_{i}, A\right\rangle=0
$$

Since $A$ is positive semidefinite, we can write $A=T^{2}$ for some symmetric $n \times n$ matrix $T$.

Let us fix the standard Gaussian probability measure $\mu_{n}$ in $\mathbb{R}^{n}$ with density

$$
\frac{1}{(2 \pi)^{n / 2}} e^{-\|x\|^{2} / 2}
$$

and let $x \in \mathbb{R}^{n}$ be a random vector. Then

$$
\mathbf{E}\|T x\|^{2}=\mathbf{E}\langle T x, T x\rangle=\mathbf{E}\left\langle T^{2} x, x\right\rangle=\operatorname{trace}\left(T^{2}\right)=\operatorname{trace}(A)=1 .
$$

Hence by Part (2) of Lemma 2.1,

$$
\mathbf{P}\left(x:\|T x\|^{2} \geq 6\right) \leq \phi(6)<0.07
$$

(choosing $\alpha=3$ in the definition of $\phi(6)$, we obtain $\phi(6) \leq 5 / 72<0.07$ ).

Furthermore,

$$
\begin{aligned}
\mathbf{E} q_{i}(T x) & =\left\langle Q_{i} T x, T x\right\rangle=\left\langle T Q_{i} T x, x\right\rangle=\operatorname{trace}\left(T Q_{i} T\right) \\
& =\operatorname{trace}\left(Q_{i} T^{2}\right)=\left\langle Q_{i}, A\right\rangle=1 \text { for } i=1, \ldots, k .
\end{aligned}
$$

Therefore, by Part (1) of Lemma 2.1,

$$
\mathbf{E}\left|\ln q_{i}(T x)\right| \leq 2.75 \quad \text { for } \quad i=1, \ldots, k
$$

and hence

$$
\mathbf{E}\left|\sum_{i=1}^{k} \alpha_{i} \ln q_{i}(T x)\right| \leq 2.75
$$


Therefore, by the Markov inequality,

$$
\mathbf{P}\left(x: \quad \sum_{i=1}^{k} \alpha_{i} \ln q_{i}(T x) \leq-3\right) \leq \frac{2.75}{3}<0.92
$$

From (3.2.2)-(3.2.3) we conclude that there is an $x \in \mathbb{R}^{n} \backslash\{0\}$ such that

$$
\|T x\|^{2}<6 \text { and } \sum_{i=1}^{k} \alpha_{i} \ln q_{i}(T x)>-3 .
$$

Then for

$$
y=\frac{T x}{\|T x\|}
$$

we have

$$
y \in \mathbb{S}^{n-1} \quad \text { and } \quad \sum_{i=1}^{k} \alpha_{i} \ln q_{i}(y)>-3-\ln (6)>-4.8,
$$

and, in view of (3.2.1), the proof follows.

\section{Proof of Theorem 1.1}

Proof. Let us write

$$
q_{i}(x)=\left\langle Q_{i}, x \otimes x\right\rangle \quad \text { for } \quad i=1, \ldots, k,
$$

where $Q_{1}, \ldots, Q_{k}$ are $n \times n$ positive definite matrices. Let

$$
S=\sum_{i=1}^{k} Q_{i}
$$

Thus $S \succ 0$ and hence there exists an invertible symmetric matrix $T: \mathbb{R}^{n} \longrightarrow \mathbb{R}^{n}$ such that $S=T^{2}$. Let us define new matrices

$$
\widehat{Q}_{i}=T^{-1} Q_{i} T^{-1} \quad \text { for } \quad i=1, \ldots, k,
$$

the corresponding quadratic forms

$$
\widehat{q}_{i}(x)=\left\langle\widehat{Q}_{i}, x \otimes x\right\rangle=\left\langle Q_{i}, T^{-1} x \otimes T^{-1} x\right\rangle=q_{i}\left(T^{-1} x\right) \quad \text { for } \quad i=1, \ldots, k
$$

and the map $\widehat{\psi}: \mathbb{R}^{n} \longrightarrow \mathbb{R}^{k}$,

$$
\widehat{\psi}(x)=\left(\widehat{q}_{1}, \ldots, \widehat{q}_{k}\right)
$$


Clearly, $\psi\left(\mathbb{R}^{n}\right)=\widehat{\psi}\left(\mathbb{R}^{n}\right)$ and

$$
\sum_{i=1}^{k} \widehat{Q}_{i}=I
$$

Hence, without loss of generality, we can assume that

$$
\sum_{i=1}^{k} Q_{i}=I
$$

Since $a \in \operatorname{conv}\left(\psi\left(\mathbb{R}^{n}\right)\right)$, we can write

$$
a_{i}=\left\langle Q_{i}, X\right\rangle \quad \text { for } \quad i=1, \ldots, k
$$

and some $X \succeq 0$. Moreover, in view of (4.1), we have

$$
1=\sum_{i=1}^{k} a_{i}=\left\langle\sum_{i=1}^{k} Q_{i}, X\right\rangle=\langle I, X\rangle=\operatorname{trace}(X) .
$$

We note that

$$
\sum_{i=1}^{k} a_{i} \ln \left\langle Q_{i}, X\right\rangle=\sum_{i=1}^{k} a_{i} \ln a_{i}
$$

By Theorem 3.2, there is an $x \in \mathbb{S}^{n-1}$ such that

$$
\beta+\sum_{i=1}^{k} a_{i} \ln q_{i}(x) \geq \sum_{i=1}^{k} a_{i} \ln a_{i} .
$$

Letting

$$
b_{i}=q_{i}(x) \quad \text { for } \quad i=1, \ldots, k,
$$

we conclude that

$$
\sum_{i=1}^{k} b_{i}=\sum_{i=1}^{k}\left\langle Q_{i}, x \otimes x\right\rangle=\langle I, x \otimes x\rangle=\operatorname{trace}(x \otimes x)=1
$$

and that

$$
\sum_{i=1}^{k} a_{i} \ln \left(\frac{a_{i}}{b_{i}}\right)=\sum_{i=1}^{k} a_{i} \ln a_{i}-\sum_{i=1}^{k} a_{i} \ln b_{i} \leq \beta .
$$

Moreover, for $b=\left(b_{1}, \ldots, b_{k}\right)$ we have $b=\psi(x)$, so $b \in \psi\left(\mathbb{R}^{n}\right)$. 


\section{Proof of Theorem 1.2}

(5.1) Lemma. For a positive integer $m$ let us consider $\mathbb{R}^{m n}$ as the direct sum

$$
\mathbb{R}^{m n}=\underbrace{\mathbb{R}^{n} \oplus \ldots \oplus \mathbb{R}^{n}}_{m \text { times }} .
$$

Let us fix the standard Gaussian probability measure $\mu_{n}$ in $\mathbb{R}^{n}$ and consider the standard Gaussian probability measure $\mu_{m n}$ in $\mathbb{R}^{m n}$ as the direct product

$$
\mu_{m n}=\mu_{n} \otimes \ldots \otimes \mu_{n}
$$

Let $q: \mathbb{R}^{n} \longrightarrow \mathbb{R}$ be a positive semidefinite quadratic form and let us define a quadratic form $q_{m}: \mathbb{R}^{m n} \longrightarrow \mathbb{R}$ by

$$
q_{m}\left(x_{1}, \ldots, x_{m}\right)=\frac{1}{m} \sum_{i=1}^{m} q\left(x_{i}\right) \quad \text { where } \quad x=\left(x_{1}, \ldots, x_{m}\right)
$$

and $x_{i} \in \mathbb{R}^{n}$ for $i=1, \ldots, m$. Suppose that

$$
\mathbf{E} q=1 .
$$

Then

(1) For all $t \geq 1$ we have

$$
\mathbf{P}\left(x \in \mathbb{R}^{m n}: q_{m}(x) \geq t\right) \leq \exp \left\{\frac{m}{2}(1-t+\ln t)\right\} ;
$$

(2) For all $0<t \leq 1$ we have

$$
\mathbf{P}\left(x \in \mathbb{R}^{m n}: q_{m}(x) \leq t\right) \leq \exp \left\{\frac{m}{2}(1-t+\ln t)\right\} ;
$$

(3) We have

$$
\mathbf{E}\left|\ln q_{m}\right| \leq \frac{6}{\sqrt{m}}
$$

Proof. We use the Laplace transform method, see also [HW71]. Since

$$
\mathbf{E} q=1,
$$

in some orthonormal basis of $\mathbb{R}^{n}$ we can write

$$
q(x)=\sum_{i=1}^{n} \lambda_{i} \xi_{i}^{2} \quad \text { where } \quad x=\left(\xi_{1}, \ldots, \xi_{n}\right)
$$


and

$$
\sum_{i=1}^{n} \lambda_{i}=1 \quad \text { and } \quad \lambda_{i} \geq 0 \quad \text { for } \quad i=1, \ldots, n .
$$

Writing vectors $x \in \mathbb{R}^{m n}$ as $x=\left(\xi_{11}, \ldots, \xi_{1 n}, \xi_{21}, \ldots, \xi_{2 n}, \ldots, \xi_{m 1}, \ldots, \xi_{m n}\right)$, we write

$$
q_{m}(x)=\frac{1}{m} \sum_{i=1}^{n} \sum_{j=1}^{m} \lambda_{i} \xi_{j i}^{2}
$$

For any $0<\alpha<m / 2$ we have

$$
\begin{aligned}
\mathbf{P}\left(x \in \mathbb{R}^{m n}: q_{m}(x) \geq t\right) & =\mathbf{P}\left(x \in \mathbb{R}^{m n}: e^{\alpha q_{m}(x)} \geq e^{\alpha t}\right) \leq e^{-\alpha t} \mathbf{E} e^{\alpha q_{m}} \\
& =e^{-\alpha t} \prod_{i=1}^{n}\left(1-\frac{2 \alpha \lambda_{i}}{m}\right)^{-m / 2}
\end{aligned}
$$

Since the function

$$
\left(\lambda_{1}, \ldots, \lambda_{n}\right) \longmapsto-\frac{m}{2} \sum_{i=1}^{n} \ln \left(1-\frac{2 \alpha \lambda_{i}}{m}\right)
$$

is convex, it attains its maximum on the simplex (5.1.1) at a vertex $\lambda_{i}=1, \lambda_{j}=0$ for $j \neq i$. Therefore,

$$
\mathbf{P}\left(x \in \mathbb{R}^{m n}: q_{m}(x) \geq t\right) \leq e^{-\alpha t}\left(1-\frac{2 \alpha}{m}\right)^{-m / 2}
$$

Optimizing on $\alpha$, we choose

$$
\alpha=\frac{m}{2}\left(\frac{t-1}{t}\right)
$$

and the proof of Part (1) follows.

For any $\alpha>0$ we have

$$
\begin{aligned}
\mathbf{P}\left(x \in \mathbb{R}^{m n}: q_{m}(x) \leq t\right) & =\mathbf{P}\left(x \in \mathbb{R}^{m n}: e^{-\alpha q_{m}(x)} \geq e^{-\alpha t}\right) \leq e^{\alpha t} \mathbf{E} e^{-\alpha q_{m}} \\
& =e^{\alpha t} \prod_{i=1}^{n}\left(1+\frac{2 \alpha \lambda_{i}}{m}\right)^{-m / 2} .
\end{aligned}
$$

Since the function

$$
\left(\lambda_{1}, \ldots, \lambda_{n}\right) \longmapsto-\frac{m}{2} \sum_{i=1}^{n} \ln \left(1+\frac{2 \alpha \lambda_{i}}{m}\right)
$$


is convex, it attains its maximum on the simplex (5.1.1) at a vertex $\lambda_{i}=1, \lambda_{j}=0$ for $j \neq i$. Therefore,

$$
\mathbf{P}\left(x \in \mathbb{R}^{m n}: q_{m}(x) \leq t\right) \leq e^{\alpha t}\left(1+\frac{2 \alpha}{m}\right)^{-m / 2}
$$

Optimizing on $\alpha$, we choose

$$
\alpha=\frac{m}{2}\left(\frac{1-t}{t}\right)
$$

and the proof of Part (2) follows.

Let us define

$$
X_{+}=\left\{x \in \mathbb{R}^{m n}: q_{m}(x) \geq 1\right\} \quad \text { and } \quad X_{-}=\left\{x \in \mathbb{R}^{m n}: q_{m}(x)<1\right\}
$$

Then

$$
\mathbf{E}\left|\ln q_{m}\right|=\int_{X_{+}} \ln q_{m}(x) d \mu_{m n}(x)-\int_{X_{-}} \ln q_{m}(x) d \mu_{m n}(x)
$$

By Part (1),

$$
\begin{aligned}
\int_{X_{+}} \ln q_{m}(x) d \mu_{m n}(x) & =\int_{0}^{+\infty} \mathbf{P}\left(x: \ln q_{m}(x) \geq t\right) d t \\
& =\int_{0}^{+\infty} \mathbf{P}\left(x: q_{m}(x) \geq e^{t}\right) d t \\
& \leq \int_{0}^{+\infty} \exp \left\{\frac{m}{2}\left(1-e^{t}+t\right)\right\} d t \leq \int_{0}^{+\infty} \exp \left\{-\frac{m t^{2}}{4}\right\} d t \\
& =\sqrt{\frac{\pi}{m}} .
\end{aligned}
$$

By Part(2),

$$
\begin{aligned}
\int_{X_{-}}-\ln q_{m}(x) d \mu_{m n}(x) & =\int_{0}^{+\infty} \mathbf{P}\left(x:-\ln q_{m}(x) \geq t\right) d t \\
& =\int_{0}^{+\infty} \mathbf{P}\left(x: q_{m}(x) \leq e^{-t}\right) d t \\
& \leq \int_{0}^{+\infty} \exp \left\{\frac{m}{2}\left(1-e^{-t}-t\right)\right\} d t .
\end{aligned}
$$


Now,

$$
\begin{aligned}
\int_{0}^{+\infty} \exp \left\{\frac{m}{2}\left(1-e^{-t}-t\right)\right\} d t & =\int_{0}^{1} \exp \left\{\frac{m}{2}\left(1-e^{-t}-t\right)\right\} d t \\
& +\int_{1}^{+\infty} \exp \left\{\frac{m}{2}\left(1-e^{-t}-t\right)\right\} d t \\
& \leq \int_{0}^{1} \exp \left\{-\frac{m t^{2}}{6}\right\} d t+\int_{0}^{+\infty} \exp \left\{-\frac{m t}{2}\right\} d t \\
& \leq \sqrt{\frac{3 \pi}{2 m}}+\frac{2}{m}
\end{aligned}
$$

Summarizing,

$$
\mathbf{E}\left|\ln q_{m}\right| \leq \sqrt{\frac{\pi}{m}}+\sqrt{\frac{3 \pi}{2 m}}+\frac{2}{m}<\frac{6}{\sqrt{m}}
$$

and the proof of Part (3) follows.

(5.2) Theorem. Let $\alpha_{1}, \ldots, \alpha_{k}$ be non-negative reals such that $\alpha_{1}+\ldots+\alpha_{k}=1$, let $Q_{1}, \ldots, Q_{k}$ be $n \times n$ positive definite matrices and let $m$ be a positive integer. Then

$$
\begin{gathered}
\max _{\substack{X \succeq 0 \\
\operatorname{trace}(X)=1 \\
\operatorname{rank} X \leq m}} \sum_{i=1}^{k} \alpha_{i} \ln \left\langle Q_{i}, X\right\rangle \leq \max _{\substack{X \succeq 0 \\
\operatorname{trace}(X)=1}} \sum_{i=1}^{k} \alpha_{i} \ln \left\langle Q_{i}, X\right\rangle \\
\leq \frac{15}{\sqrt{m}}+\max _{\substack{X \succeq 0 \\
\operatorname{trace}(X)=1 \\
\operatorname{rank}(X) \leq m}} \sum_{i=1}^{k} \alpha_{i} \ln \left\langle Q_{i}, X\right\rangle .
\end{gathered}
$$

Proof. The first inequality obviously holds.

Let $A$ be a matrix where the maximum value of the function

$$
X \longmapsto \sum_{i=1}^{k} \alpha_{i} \ln \left\langle Q_{i}, X\right\rangle
$$

is attained on the set $X$ of positive semidefinite matrices of trace 1. Rescaling $Q_{i} \longrightarrow \tau_{i} Q_{i}$ for some positive $\tau_{1}, \ldots, \tau_{k}$ if necessary, we may assume that $\left\langle Q_{i}, A\right\rangle=$ 1 for $i=1, \ldots, k$ and hence

$$
\max _{\substack{X \succeq 0 \\ \operatorname{trace}(X)=1}} \sum_{i=1}^{k} \alpha_{i} \ln \left\langle Q_{i}, X\right\rangle=\sum_{i=1}^{k} \alpha_{i} \ln \left\langle Q_{i}, A\right\rangle=0
$$


Since $A$ is positive semidefinite, we can write $A=T^{2}$ for some symmetric $n \times n$ matrix $T$.

Let us fix the standard Gaussian probability measure $\mu_{n}$ in $\mathbb{R}^{n}$ with density

$$
\frac{1}{(2 \pi)^{n / 2}} e^{-\|x\|^{2} / 2}
$$

and let $x_{1}, \ldots, x_{m} \in \mathbb{R}^{n}$ be $m$ independent random vectors. Then

$$
\mathbf{E}\left\|T x_{j}\right\|^{2}=\mathbf{E}\left\langle T x_{j}, T x_{j}\right\rangle=\mathbf{E}\left\langle T^{2} x_{j}, x_{j}\right\rangle=\operatorname{trace}\left(T^{2}\right)=\operatorname{trace}(A)=1 .
$$

Applying Part (1) of Lemma 5.2, we conclude that

$$
\mathbf{P}\left(x_{1}, \ldots, x_{m}: \frac{1}{m} \sum_{j=1}^{m}\left\|T x_{j}\right\|^{2} \geq 1+\frac{3}{\sqrt{m}}\right) \leq \exp \left\{-\frac{9}{8}\right\}<0.33
$$

(we use that $\ln (1+s) \leq s-s^{2} / 4$ for $0 \leq s \leq 1$ ).

Let us define quadratic forms

$$
q_{i}(x)=\left\langle Q_{i}, x \otimes x\right\rangle \quad \text { for } \quad i=1, \ldots, k \text {. }
$$

Then

$$
\begin{aligned}
\mathbf{E} q_{i}\left(T x_{j}\right) & =\left\langle Q_{i} T x_{j}, T x_{j}\right\rangle=\left\langle T Q_{i} T x_{j}, x_{j}\right\rangle=\operatorname{trace}\left(T Q_{i} T\right) \\
& =\operatorname{trace}\left(Q_{i} T^{2}\right)=\left\langle Q_{i}, A\right\rangle=1 \quad \text { for } \quad i=1, \ldots, k
\end{aligned}
$$

Therefore, by Part (3) of Lemma 5.1,

$$
\mathbf{E}\left|\ln \left(\frac{1}{m} \sum_{j=1}^{m} q_{i}\left(T x_{j}\right)\right)\right| \leq \frac{6}{\sqrt{m}} \text { for } i=1, \ldots, k
$$

and hence

$$
\mathbf{E}\left|\sum_{i=1}^{k} \alpha_{i} \ln \left(\frac{1}{m} \sum_{j=1}^{m} q_{i}\left(T x_{j}\right)\right)\right| \leq \frac{6}{\sqrt{m}} .
$$

Therefore, by the Markov inequality,

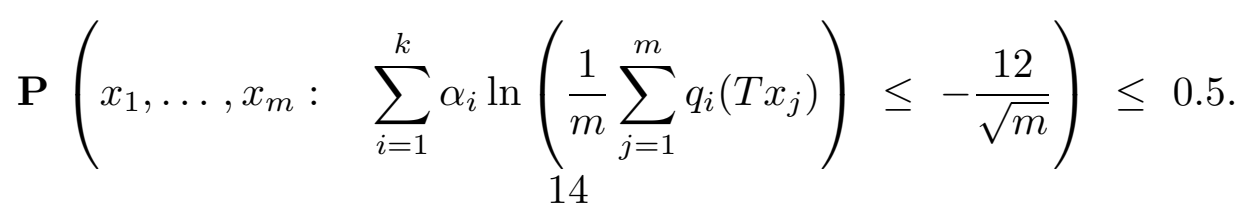


From (5.2.2)-(5.2.3) we conclude that there are points $x_{1}, \ldots, x_{m} \in \mathbb{R}^{n} \backslash\{0\}$ such that

$$
\frac{1}{m} \sum_{j=1}^{m}\left\|T x_{j}\right\|^{2} \leq 1+\frac{3}{\sqrt{m}} \text { and } \sum_{i=1}^{k} \alpha_{i} \ln \left(\frac{1}{m} \sum_{j=1}^{m} q_{i}\left(T x_{j}\right)\right) \geq-\frac{12}{\sqrt{m}} .
$$

Let us define a matrix $Y$ by

$$
Y=\left(\sum_{j=1}^{m}\left\|T x_{j}\right\|^{2}\right)^{-1} \sum_{j=1}^{m}\left(T x_{j}\right) \otimes\left(T x_{j}\right) .
$$

Then

$$
Y \succeq 0, \quad \operatorname{trace}(Y)=1, \quad \operatorname{rank} Y \leq m
$$

and

$$
\begin{gathered}
\sum_{i=1}^{k} \alpha_{i} \ln \left\langle Q_{i}, Y\right\rangle=\sum_{i=1}^{k} \alpha_{i} \ln \left(\frac{1}{m} \sum_{j=1}^{m} q_{i}\left(x_{j}\right)\right)-\ln \left(\frac{1}{m} \sum_{j=1}^{m}\left\|T x_{j}\right\|^{2}\right) \\
\geq-\frac{12}{\sqrt{m}}-\ln \left(1+\frac{3}{\sqrt{m}}\right)>-\frac{15}{\sqrt{m}}
\end{gathered}
$$

and, in view of (5.2.1), the proof follows.

(5.3) Proof of Theorem 1.2. As in the proof of Theorem 1.1 in Section 4, without loss of generality we assume that

$$
\sum_{i=1}^{k} Q_{i}=I
$$

Since $a \in \operatorname{conv}\left(\psi\left(\mathbb{R}^{n}\right)\right)$, we can write

$$
a_{i}=\left\langle Q_{i}, X\right\rangle \quad \text { for } \quad i=1, \ldots, k
$$

and some $X \succeq 0$. Moreover, in view of (5.3.1), we have

$$
1=\sum_{i=1}^{k} a_{i}=\left\langle\sum_{i=1}^{k} Q_{i}, X\right\rangle=\langle I, X\rangle=\operatorname{trace}(X)
$$

We note that

$$
\sum_{i=1}^{k} a_{i} \ln \left\langle Q_{i}, X\right\rangle=\sum_{i=1}^{k} a_{i} \ln a_{i}
$$


By Theorem 5.2, there is a $n \times n$ symmetric matrix $Y$, such that $Y \succeq 0, \operatorname{rank} Y \leq m$ and

$$
\frac{15}{\sqrt{m}}+\sum_{i=1}^{k} a_{i} \ln \left\langle Q_{i}, Y\right\rangle>\sum_{i=1}^{k} a_{i} \ln a_{i} .
$$

Let

$$
b_{i}=\left\langle Q_{i}, Y\right\rangle \quad \text { for } \quad i=1, \ldots, k \text {. }
$$

Then

$$
\sum_{i=1}^{k} b_{i}=\left\langle\sum_{i=1}^{k} Q_{i}, Y\right\rangle=\operatorname{trace}(Y)=1 .
$$

Since $\operatorname{rank} Y \leq m$, we can write

$$
Y=\frac{1}{m} \sum_{j=1}^{m} y_{j} \otimes y_{j}
$$

for some $y_{1}, \ldots, y_{m} \in \mathbb{R}^{n}$. Then

$$
b_{i}=\frac{1}{m} \sum_{j=1}^{m} q_{i}\left(y_{j}\right) \quad \text { for } \quad i=1, \ldots, k
$$

and $b$ is a convex combination of at most $m$ points from $\psi\left(\mathbb{R}^{n}\right)$.

\section{ACKNOWLEDGMENT}

I am grateful to Roman Vershynin for several useful suggestions and references.

\section{REFERENCES}

[Ba99] A. Barvinok, Polynomial time algorithms to approximate permanents and mixed discriminants within a simply exponential factor, Random Structures \& Algorithms 14 (1999), 29-61.

[Ba02] A. Barvinok, A Course in Convexity, Graduate Studies in Mathematics, vol. 54, American Mathematical Society, Providence, RI, 2002.

[CT06] T.M. Cover and J.A. Thomas, Elements of Information Theory, Second edition, WileyInterscience [John Wiley \& Sons], Hoboken, NJ, 2006.

[HW71] D.L. Hanson and F.T. Wright, A bound on tail probabilities for quadratic forms in independent random variables, Ann. Math. Statist. 42 (1971), 1079-1083.

[Ma08] J. Matoušek, On variants of the Johnson - Lindenstrauss lemma, Random Structures \& Algorithms 33 (2008), 142-156.

[Pi81] G. Pisier, Remarques sur un résultat non publié de B. Maurey, Seminar on Functional Analysis, 1980-1981, Exp. No. V, 13 pp. (1981), École Polytech., Palaiseau.

[PT07] I. Pólik and T. Terlaky, A survey of the S-lemma, SIAM Rev. 49 (2007), 371-418.

[Tu10] L. Tunçel, Polyhedral and Semidefinite Programming Methods in Combinatorial Optimization, Fields Institute Monographs, 27, American Mathematical Society, Providence, RI; Fields Institute for Research in Mathematical Sciences, Toronto, ON, 2010.

[Ve+] R. Vershynin, Lectures in Geometric Functional Analysis, Book in progress, available at http://www-personal.umich.edu/ romanv/papers/GFA-book/GFA-book.pdf.

Department of Mathematics, University of Michigan, Ann Arbor, Mi 48109-1043, USA

E-mail address: barvinok@umich.edu 